\title{
Direct Sampling in Multi-channel Synchronous TDEMI Measurements
}

\author{
Tom Hartman*, Niek Moonen*, Frank Leferink*† \\ *University of Twente, Enschede, Netherlands \\ ${ }^{\dagger}$ Thales Nederland B.V., Hengelo, Netherlands \\ tom.hartman@utwente.nl
}

\begin{abstract}
This paper shows possible benefits of multi-channel synchronous time-domain electromagnetic interference (TDEMI) measurements. The setup was developed with respect to lowfrequency conducted Electromagnetic Interference (EMI) measurements in high power, fast switching systems using a lowcost solution. Using an 8-channel digitizer voltages, currents and magnetic fields were simultaneously recorded. Using digital signal post-processing investigations were performed on the relation between switching currents and magnetic fields, while also investigating the time variance of the load impedance.
\end{abstract}

\section{INTRODUCTION}

EMI has been traditionally analyzed based on its spectral content. Due to the limitations in hardware, a time domain analysis was insufficiently accurate. Either due to the limited Analog to Digital Converter (ADC), sampling rate, memory, or dynamic range [1]. Using an EMI test receiver, overcame these issues by analyzing each frequency bin/band individually while sweeping through the spectrum. Inherently, the switch to super-heterodyne receiver introduced trade-offs. Measurement times increased, while time domain information was lost in the peak detector. Therefore correctly analyzing the disturbance requires the disturbance to be repetitive in nature, while maintaining a constant amplitude. Different types of detectors addressed this issue. A good explanation of analyzing time variant disturbances can be found in [2], since a simulation model is developed to mimic different types of detectors. To summarize, issues with super-heterodyne receivers are often related to the type of the disturbance being unknown.

- narrowband vs broadband

- continues wave vs transient

- time-consuming

With the recent development of fast Fourier transform (FFT) based Receivers, EMI measurements have become much easier. Several TDEMI measurement techniques have been studied [3]-[5]. Digital decomposition of Common Mode (CM) and Differential Mode (DM) has been shown in for instance [3], [6], which requires a multi-channel approach. Transient decomposition is shown in [7] using short time fast Fourier transform (STFFT) techniques. While in [4], [8] it is proposed to use STFFT techniques to present timefrequency plots (i.e. spectrograms), however engineers need to be trained in analyzing these results, and standards still need to be developed. However the biggest drawback, is that the Commercial of the Shelf (COTS) available FFT-receivers are single input with a heterodyne chain.

With the development of low-cost digitizers, the possibility for creating ones own low-cost receiver arises. It has for instance been used in developing a cost effective and fast magnetic emission test platform [9].

This paper will focus on applications of multi-channel synchronous measurements as stated in [5] and the possible challenges it brings. In [6] it was applied to decompose, and compare multiple measurement techniques. This paper will show the possibility to investigate magnetic emissions related to switching transients, and also the possibility to analyze a time variant impedance. In either case, a synchronous multi-channel solution is required. An 8-channel digitizer has been used to record the phase-, neutral- and DM- current and voltages. Additionally the magnetic field has also been recorded according to the RE101 standard.

The general overview of the total measurement can be found in [6], which focuses on the conducted part of the measurement. The 8-channel scope is used in conjunction with the 'mains monitor box' as described in [10] and will be elaborated on in Section III. Voltage and current measurements have an overlapping bandwidth from approximately $2 \mathrm{kHz}$ to $100 \mathrm{kHz}$. Next to conducted measurements, the radiating magnetic field is recorded to investigate its relation to fast switching currents. The results presented in section IV are qualitatively reviewed, as this is a proof-of-concept type of setup.

\section{THEORETICAL BACKGROUND}

The multi-channel approach allows for exploration in the unknown and novel domains at relative low costs. As was explained, in this paper we aim for the current to magnetic relationship, and the concept of a time varying impedance. In the following subsections, the concepts are further explored and the motivation for exploration is given.

\section{A. Magnetic Field}

As is well known from the Maxwell equations, a current induces a magnetic field and furthermore magnetic fields induce currents as is explained by Lenz's law. We measure magnetic fields with loop antennas, which act as transducers for magnetic flux. For instance an output voltage of the antenna 


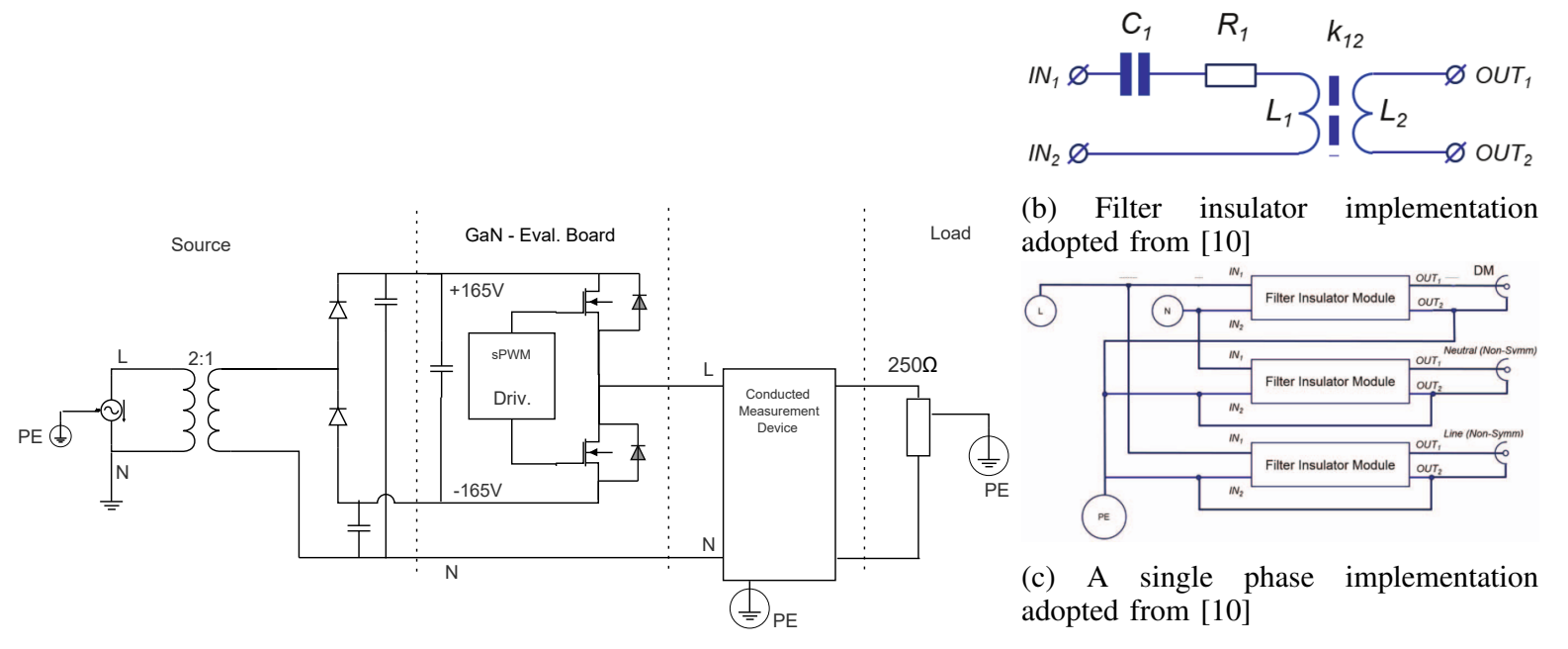

(a) setup

Fig. 1: Schematic representation of the test setup [6]

is created by means of a varying magnetic flux, which can be seen using the following equations:

$$
\begin{gathered}
\mathcal{E}=-\frac{d \Phi}{d t}, \quad(1) \quad \Phi=\iint_{S} \mathbf{B} \cdot d A \\
\oint \mathbf{B} \cdot d \mathbf{l}=\mu_{0} I_{e n c}
\end{gathered}
$$

From combining equations 1,2 and 3 we can see a proportional relationship exists between the time-derivative of the current inside a system and the measured magnetic field, i.e. the voltage measured by the antenna is proportional to the first order time-derivative of the current in the system, as seen in equation 4:

$$
\mathbf{B} \propto \frac{d i}{d t}
$$

In the measurement setup, current and magnetic field have both been recorded simultaneously. This allows for an investigation into the radiation transfer function (radiation efficiency) of the entire system. The relation between E-field and varying voltage has been researched in [11]. Following the same assumption of a Linear Time-Invariant (LTI) system shown in that paper, a possible attenuation profile might be derived. However the amount of measurements in the current setup is limited and since the focus of this paper is on the multichannel low cost digitizer possibilities, a full study of transfer function of the system is regarded to be future work.

The Digital Signal Processing (DSP) done in this work, is related to the MIL-STD RE101. We want to examine the relation between switching events and B-fields in time, however the recorded signal is always a time varying voltage. The recorded signal $\mathrm{x}(\mathrm{t})$, should first be transformed into a spectrum in $\mathrm{dBuV}$, to which the antenna factor can be applied. From this the time variant magnetic field can be recovered via the inverse Fourier transform, seen in equations 5, 6 and 7 respectively.

$$
\begin{gathered}
\mathcal{F}[x(t)]=X(\omega), \quad(5) \quad Y(\omega)=X(\omega) \cdot A F(\omega) \\
\mathcal{F}^{-1}[(Y(\omega))]=y(t)
\end{gathered}
$$

With $x(t)$ being the recorded voltage from the loop antenna, $y(t)$ the magnetic field measured, and AF the antenna factor. Performing these operations will result in a complex valued time series, of which only the real values have meaning and its unit will be in pT. The imaginary part is a result of rounding errors and applying a perfect filter for frequencies above $100 \mathrm{kHz}$. Assuming a perfect symmetrical spectrum around the Nyquist frequency removes this issue. In the following subsection the concept of measuring a varying impedance is addressed.

\section{B. Varying Impedance}

The varying impedance concept originates from power grid measurements, in which many users are independently inserting and extracting loads. In case of old fashioned resistive loads, this already introduced fluctuations in the power grid.

The transition to Switch Mode Power Supply (SMPS) has introduced a more difficult task in defining a load. This can already be seen in Fig. 1, as a load of $250 \Omega$ is being used with either a positive or negative voltage. From the grid side, a constant varying $50 \mathrm{~Hz}$ sinewave is provided. However, the current drawn from the grid is either positive or negative depending on the switching state. A third state is also available, in case both of the switches can be considered to be open. This is during the time a deadtime is introduced, it is inserted to prevent the half-bridge from shorting.

Evaluating the varying impedance requires processing of the recorded voltages and currents. In the measurement setup as 
shown in Fig. 1 the voltage is recorded with a 1:10 voltage probe, while the currents are detected via a current clamp (Pico TA189). In [6] the overlapping frequency ranges were shown, in which the measurement methods are valid. In this case we assume $v(t)$ and $i(t)$ which are recorded, are also frequency dependent. Using the STFFT in both cases, the overlapping frequencies can be extracted while maintaining the time-varying information. In that case the relation in equation 8 would hold:

$$
Z(f, t)=\frac{v(f, t)}{i(f, t)}
$$

Note however that the setup, as it is shown here, will mainly address issues that arise from post-processing, as one expects to record voltages and currents running through the $250 \Omega$ load. The resulting impedance will not be the total SMPS impedance. This was done as a verification of the postprocessing.

\section{Measurement SetuP}

Safely measuring DM EMI in a relatively high voltage (i.e. above $100 \mathrm{~V}$ ) setup is done as depicted in Fig. 1a. The inside of a conducted measurement device, as seen in Fig. 1a, can be seen in Fig. 1b and Fig. 1c. As the emphasis of this paper lies in synchronous time domain measurements, the functional behavior of the AC/DC converter will only be briefly described here. The used quantities are magnetic field, DM voltage and DM current at the load.

\section{A. Galium-Nitride (GaN) Half-bridge}

In Fig. 1a it can be seen that the 'DC' source is a galvanic isolated grid that has been rectified. By using Sinusoidal Pulse Width Modulation (sPWM) driver logic, the switches are operated in such a way, that the switching node is either connected to the $+165 \mathrm{~V}$ or $-165 \mathrm{~V}$. This results in a sPWM voltage waveform that contains two main frequency components, $f_{c}$ and $f_{m}$, which are the switching frequency and AC output frequency respectively. In [11] the full background for studying a DC/AC converter with extreme flexibility in choosing these frequencies is described. However, in the evaluated setup $f_{c}=25 \mathrm{kHz}$ and $f_{m}=50 \mathrm{~Hz}$, which implies that, when the output waveform is low pass filtered, the resulting AC signal consists only of a $50 \mathrm{~Hz}$ component with an approximate $V_{r m s}=116 \mathrm{~V}$.

\section{B. Magnetic Field Measurement}

The magnetic field measurement RE101 as was shown in [9] has also been performed to the above described setup, however the time domain signal was simultaneously recorded and now available for evaluation in the results section.

\section{Varying Impedance Measurement}

This subsection is to emphasize that the extraction of the voltage and current was done according to the previous written paper [6] and is now applied to a more elaborate processing scheme that shows the benefits of using a synchronous multichannel digitizer.
Now that the measurement setup and processing of the results have been described, the results are presented in the next section.

\section{RESULTS}

The results will also be presented into two sub-sections following the structure applied throughout this paper. First the magnetic field results are shown, as this is a more predictable result. The varying impedance is a novel concept and is presented here as a possibility to investigate with a multichannel digitizer. The results shown here are only evaluated qualitatively, and quantifying the results is left to future work.

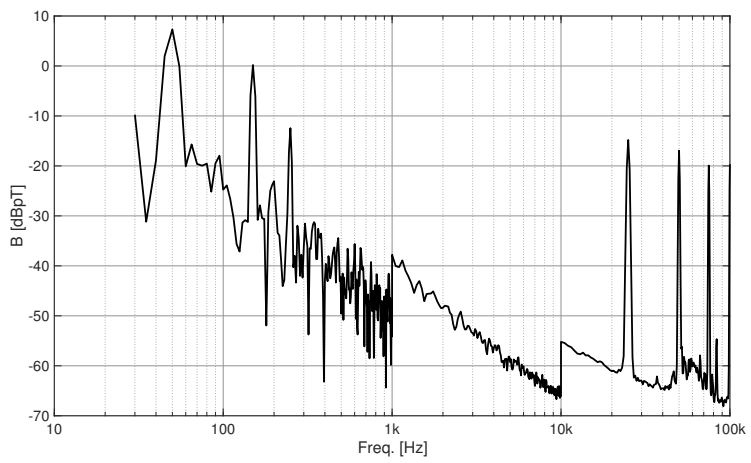

Fig. 2: Results from processing the single channel according to the RE101 standard, which is explained in [9]

\section{A. Magnetic Field results}

The measurement results are shown for the time varying magnetic field that was recorded using the standard loop antenna as is used in the MIL-STD RE101 emissions test. First the results are processed according to standard as was described in [9] and shown in Fig. 2. Emission spikes can be seen at $50 \mathrm{~Hz}, 150 \mathrm{~Hz}$ and $250 \mathrm{~Hz}$ in the lower frequency range. While in the high frequency range (above $10 \mathrm{kHz}$ ), the switching frequency of $25 \mathrm{kHz}$ and its harmonics can be seen. Note the abrupt changes in noise floor are due to the equivalent noise bandwidth that steps at the $1 \mathrm{kHz}$ and $10 \mathrm{kHz}$ frequency points from $10 \mathrm{~Hz}$ to $100 \mathrm{~Hz}$ and $100 \mathrm{~Hz}$ to $1 \mathrm{kHz}$ respectively as described in the RE101 standard. This validates the setup, working properly as intended. Now to examine the switching transients effects on the radiated emissions, Fig. 3 is created according to the procedure that was described in section II-A. The resulting waveform shown in red, shows during the current switching spikes that are either positive or negative depending on the transition state. The magnetic field is also slowly varying, which can be seen in Fig 4. In our measurement setup, the slowly varying component of the magnetic field is more significant that the magnetic field that is due to the fast switching of the GaN half-bridge. However, due to the applied processing both phenomena can be seen and evaluated if needed. 


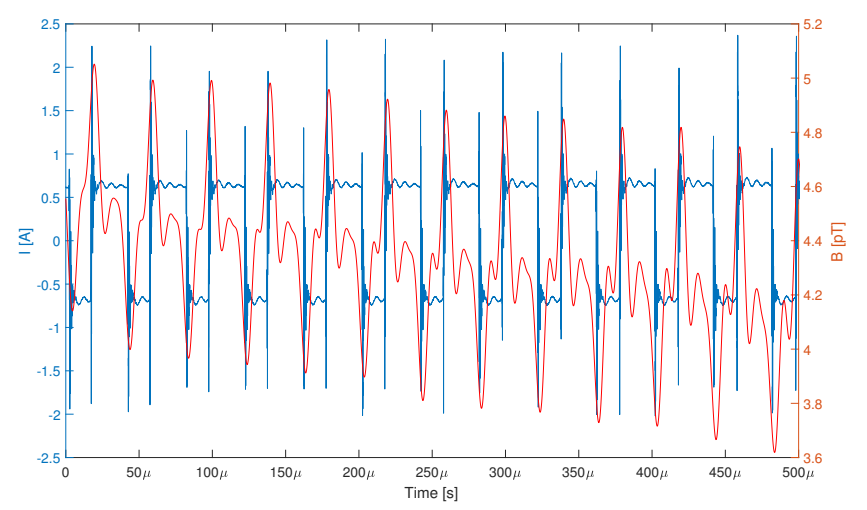

Fig. 3: The measured DM-current (blue) and magnetic field (red). The magnetic field is retrieved from the raw voltage data through DSP.

\section{B. Varying Impedance Results}

The results displayed in Fig. 5 show amplitude spectral densities that are varying in frequency as well as time. As the impedance is calculated as a ratio of voltage and currents, a very low current can result in a very high impedance. By creating a sPWM waveform, that contains the $50 \mathrm{~Hz}$ as the output, while switching at $25 \mathrm{kHz}$ only these frequency and their harmonics are measurable. At other frequencies the noise floor is being measured. This effect can be nicely seen in the upper graph in Fig. 5 and also in Fig. 6. In Fig. 6a the current is varying between $0.06 \mathrm{~A}$ and $0.09 \mathrm{~A}$, while in Fig. $6 \mathrm{~b}$ the current fluctuates between approx. $0 \mathrm{~A}$ and $0.045 \mathrm{~A}$. The resulting impedance is spiking at the moments that the current is approaching zero. From this we can conclude that the four noise bandwidths are due to the measured noise that are shown as dark blue regions, in the bottom two figures of Fig. 5.

\section{CONCLUSION}

Using an 8-channel measurement setup, multi-channel synchronous time domain measurements have been explored. Focusing on magnetic-field radiation arising from switching currents, and time variant impedances. It has also been shown that by combining low-frequency current measurements (DC$100 \mathrm{kHz})$ with high-frequency voltage measurements $(2 \mathrm{kHz}-$ $7 \mathrm{MHz}$ ), one is able to evaluate the impedance in the overlapping frequency range. Issues in calculating the impedance arise from measuring low currents, that are (in this case) due the noise in the measurement system.

As the magnetic field measurement has been performed according to RE101, the entire lower frequency range was covered $(30 \mathrm{~Hz}-100 \mathrm{kHz})$. Simultaneously the DM current was recorded in a frequency range from DC up to $100 \mathrm{kHz}$. After correction for the loop-antenna used, it was possible to see the influence of the fast switching current on the measured magnetic field. Even though the impact was lower than the slow varying component it has been shown that it is possible to benefit from multi-channel synchronous conducted TDEMI measurements applied in high power electronic systems.

\section{ACKNOWLEDGMENT}

This project has received funding from the EMPIR programme co-financed by the Participating States and from the European Union's Horizon 2020 research and innovation programme. The results found reflect the author's view only. EURAMET is not responsible for any use that may be made of the information it contains.

\section{REFERENCES}

[1] E. Puri and M. Monti, "Pitfalls in Measuring Discontinuous Disturbances with Latest Click Analysers," Emc2016, pp. 1-6, 2016.

[2] T. Karaca, B. Deutschmann, and G. Winkler, "EMI-receiver simulation model with quasi-peak detector," IEEE International Symposium on Electromagnetic Compatibility, vol. 2015-Septm, pp. 891-896, 2015.

[3] Y.-s. Lee, "Time Domain Measurement System for Conducted EMI and CM/DM Noise Signal Separation," 2005 International Conference on Power Electronics and Drives Systems, vol. 2, pp. 1640-1645, 2005. [Online]. Available: http://ieeexplore.ieee.org/lpdocs/epic03/wrapper.htm?arnumber=1619951

[4] B. J. A. M. Van Leersum, R. B. Timens, F. J. K. Buesink, and F. B. J. Leferink, "Time domain methods for the analysis of conducted interference on the power supply network of complex installations," IEEE International Symposium on Electromagnetic Compatibility, pp. 605-610, 2014

[5] M. Pous, M. Azpúrua, and F. Silva, "Benefits of Full Time-Domain EMI Measurements for Large Fixed Installation,” pp. 514-519, 2016.

[6] T. Hartman, N. Moonen, and F. Leferink, "Evaluation of Multichannel Synchronous Conducted TDEMI Measurements for High Voltage Power Electronics," in 2018 International Symposium on Electromagnetic Compatibility - EMC EUROPE, 2018, p. to be published.

[7] M. A. Azpúrua, M. Pous, and F. Silva, "Decomposition of Electromagnetic Interferences in the Time-Domain," IEEE Transactions on Electromagnetic Compatibility, vol. 58, no. 2, pp. 385-392, 2016.

[8] I. Setiawan, C. Keyer, M. Azpurua, F. Silva, and F. Leferink, "Timedomain Measurement Technique to Analyze Cyclic Short-Time Interference in Power Supply Networks," pp. 279-282, 2016.

[9] I. Setiawan, N. Moonen, F. Buesink, and F. Leferink, "Efficient Magnetic Field Measurements," 2017.

[10] C. Keyer, F. Buesink, and F. Leferink, "Mains Power Synchronous Conducted Noise Measurement in the 2 to $150 \mathrm{kHz}$ band," pp. 865869, 2016.

[11] C. V. Diemen, N. Moonen, and F. Leferink, "Estimation of Radiation Efficiency of GaN Half-bridge Based Submodule System for Radiated EMI Prediction," in 2018 International Symposium on Electromagnetic Compatibility - EMC EUROPE, 2018, p. to be published. 


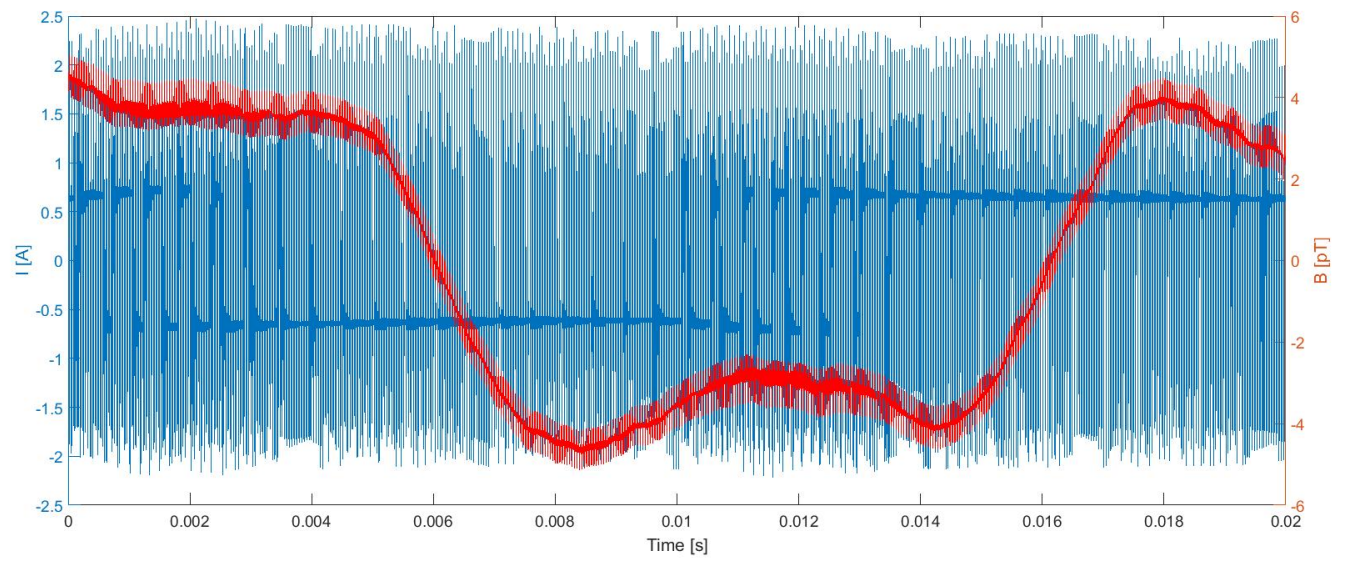

Fig. 4: The measured time signal DM-current (blue) and magnetic field (red)
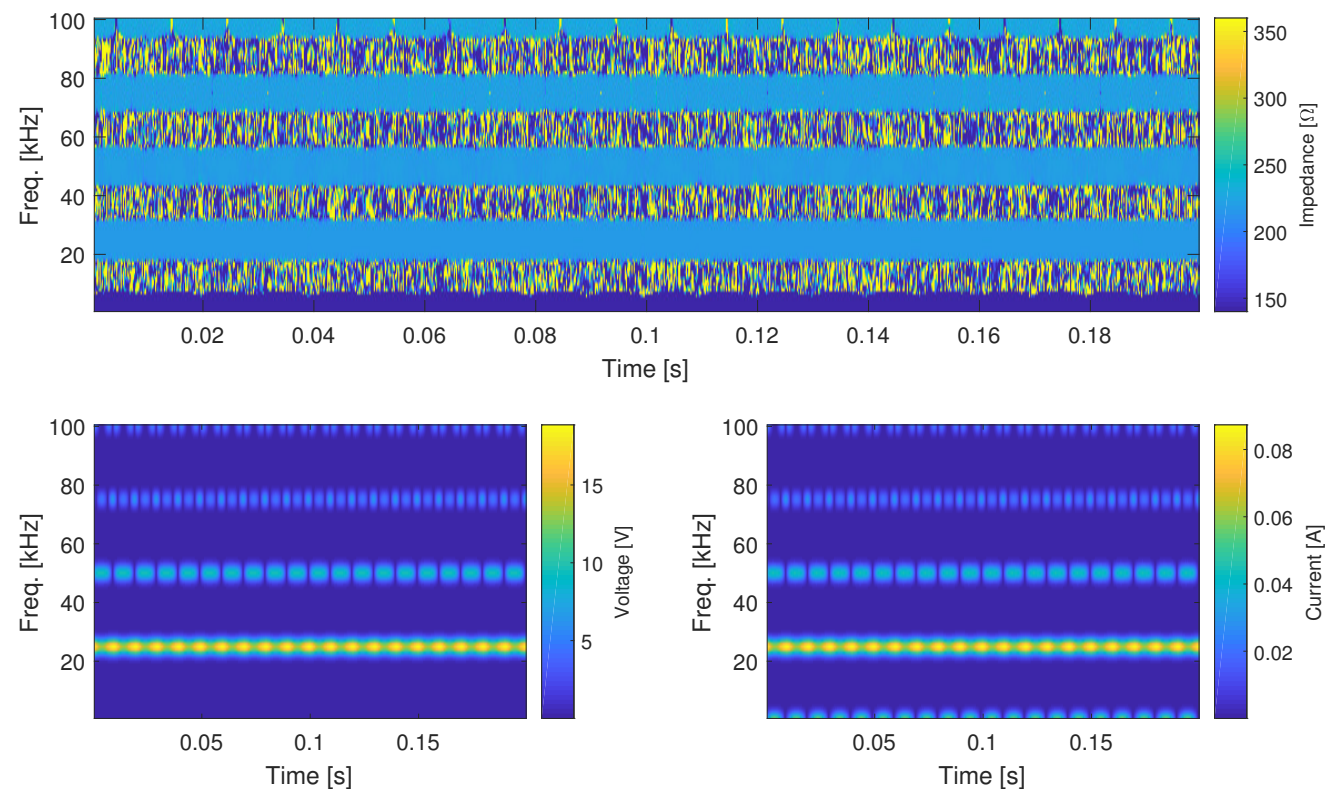

Fig. 5: The top figure displays the calculated impedance which is the ratio between the voltage STFFT and current STFFT. These are shown in the bottom figures

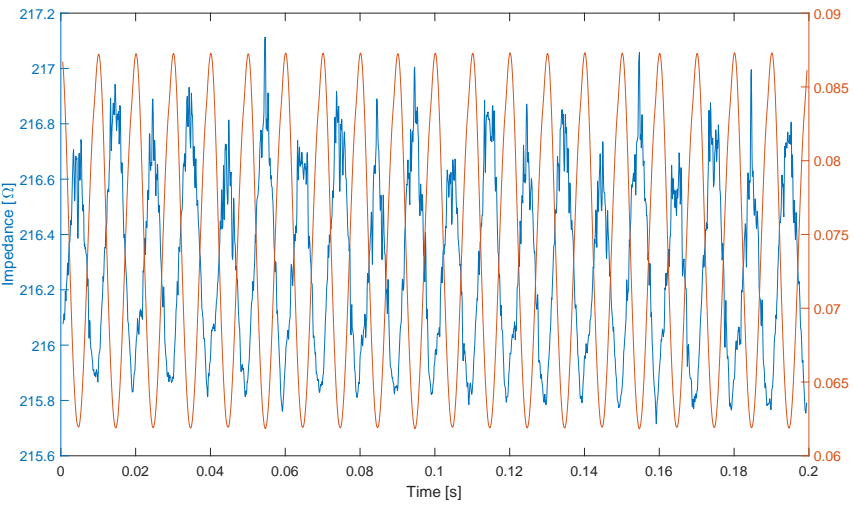

(a) Extracted impedance vs extracted current at $25 \mathrm{kHz}$

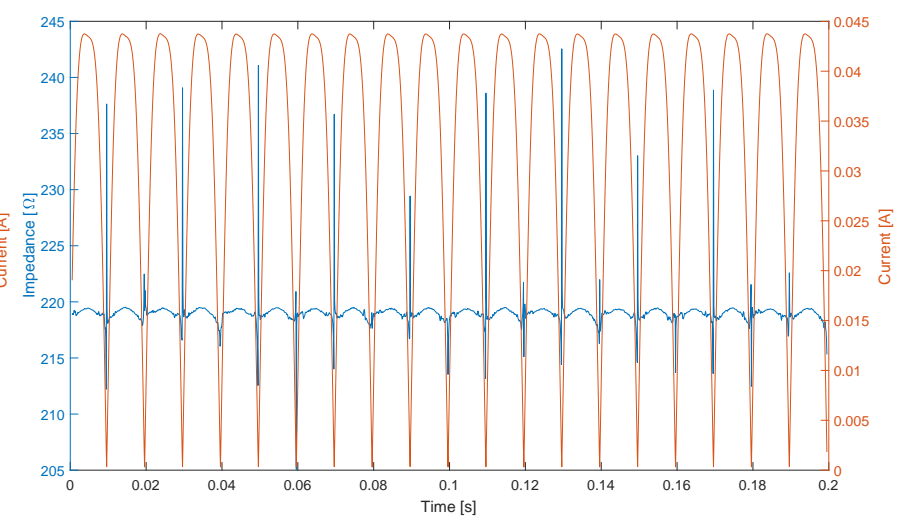

(b) Extracted impedance vs extracted current at $50 \mathrm{kHz}$

Fig. 6: The waveforms are single frequencies selected from the spectrogram displayed in Fig. 5 